\title{
Article \\ Modélisation et Identification en boucle ouverte d'un moteur à courant continu M.C.C
}

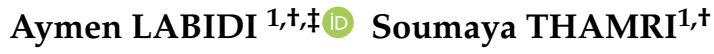 \\ 1 Affiliation: University of Carthage - National Institute of Applied Sciences and Technology - Chemical \\ Engineering Student - Chemical Engineering Department \\ + Current address: 676 INSAT Centre Urbain Nord BP Tunis Cedex 1080 \\ ‡ Aeronautical Consultant for FTAA; Glider Former and Manager for Aerobotix INSAT .
}

Citation: LABIDI, A Modélisation et Identification en boucle ouverte d'un moteur à courant continu M.C.C. Chemical Engineering 2021, 1, 0. 10.31219 /osf.io/k4egn

Published: $15^{\text {th }}$, April2021

Author's Note: You can Visit my Researchgate and Linkedin profiles for more researches.

Copyright: (C) 2021 by the author. Licensee to Aymen Labidi for Chemical Engineering - Open Resource (CC BY) license (https://creativecommons.org/licenses/by/4.0/).

\begin{abstract}
Le système SYNUM est un système numérique permettant l'étude des asservissements de mécanique ou de vitesse pour actionner un moteur à courant continu M.C.C entraînant une charge mécanique réglable et un disque gradué.

L'entreprise SYNUM a ainsi crée la première entité qui pense à la transition digitale comme une fusion synergique de l'homme et du numérique. Durant notre manipulation, utiliser le système ERD100000 qui est un MONITEUR ELECTROMECANIQUE.
\end{abstract}

Keywords: Moteur - Automatisme et régulation - Courant et Tension - Réponses - Boucle ouverte

\section{Professeure Responsable: Mme Fatma EZZAHRA OUESLATI}

\section{Objectif}

L'objectif de notre séance de travaux pratiques est d'étudier le modèle linéarisé et d'ordre réduit du moteur à courant continu. On compte opérer en boucle ouverte, c'est à dire que la commande du processus ne dépendra pas du signal de mesure issu du capteur. On va se familiariser à effectuer les essais expérimentaux permettant de déterminer les paramètres de commande.

\section{Principe}

Qu'est ce qu'un Moniteur Electromécanique ERD100000?

L'ERD100000 est un ensemble électromécanique monté sur un châssis en aluminium anodisé. Il est proposé en standard avec 2 régimes de fonctionnement :

- Pilotage par le logiciel $D_{S} y n$ (pages suivantes), en option prototypage rapide $D_{S} c i l$ (cf documentation correspondante).

- Pilotage par une station de prototypage rapide sous Matlab Simulink (carte d'interface en option non fournie), dans ce dernier cas, les entrées analogiques permettent de commander directement les interfaces de puissance, moteur, solénoïde de frottement fluide et moteur de frottement sec.

Il comprend:

- Une carte électronique de commande avec microprocesseur de haut niveau de puissance qui assure le contrôle commande en temps réel du système et la communication à un micro-ordinateur de type PC via liaison Ethernet.

- Une carte électronique de puissance qui réalise les interfaces d'alimentation du moteur (au choix courant ou tension), la commande de frottement fluide (électroaimant) et la commande de frottement sec (par motoréducteur),

- $\quad$ Des points de mesure sont disponibles en face avant sur BNC (images vitesse et position, tension et courant moteur)

- Quatre entrées analogiques. 
- Un bouton numérique de réglage de consigne externe.

L'alimentation se fait par boîtier externe.

\section{$\mathrm{Qu}^{\prime}$ est ce qu'un système SYNUM ?}

Le système SYNUM est un système numérique renfermant le pack ERD100B qui est un système didactique complet, destiné à l'étude approfondie des systèmes asservis.

Il illustre l'asservissement en vitesse ou en position d'une charge mécanique en rotation entraînée par un moteur à courant continu. Il est composé d'un moniteur électromécanique référencé ERD100000, d'un logiciel référencé ERD100100, d'un dossier technique ERD100010, de dossiers pédagogiques référencés ERD100040 ERD100060, d'accessoires (bloc alimentation, cordons de liaison), en option une extension Prototypage rapide $D_{S} c i l$.

Qu'est ce qu'un système d'asservissement ?

En automatique, un asservissement est un système dont l'objet principal est d'atteindre le plus rapidement possible sa valeur de consigne et de la maintenir, quelles que soient les perturbations externes [1]. Le principe général est de comparer la consigne et l'état du système de manière à le corriger efficacement. On parle également de système commandé par rétroaction négative ou en boucle fermée.

Qu'est ce qu'une boucle ouverte?

Une boucle ouverte est une boucle dans laquelle la commande du processus ne dépendra pas du signal de mesure issu du capteur.

Qu'est ce qu'une boucle fermée?

Une boucle fermée est une boucle dont la commande du processus est fortement liée à une intsruction de départ, un signal d'entrée imposé par l'utilisateur.

\section{Mode opératoire}

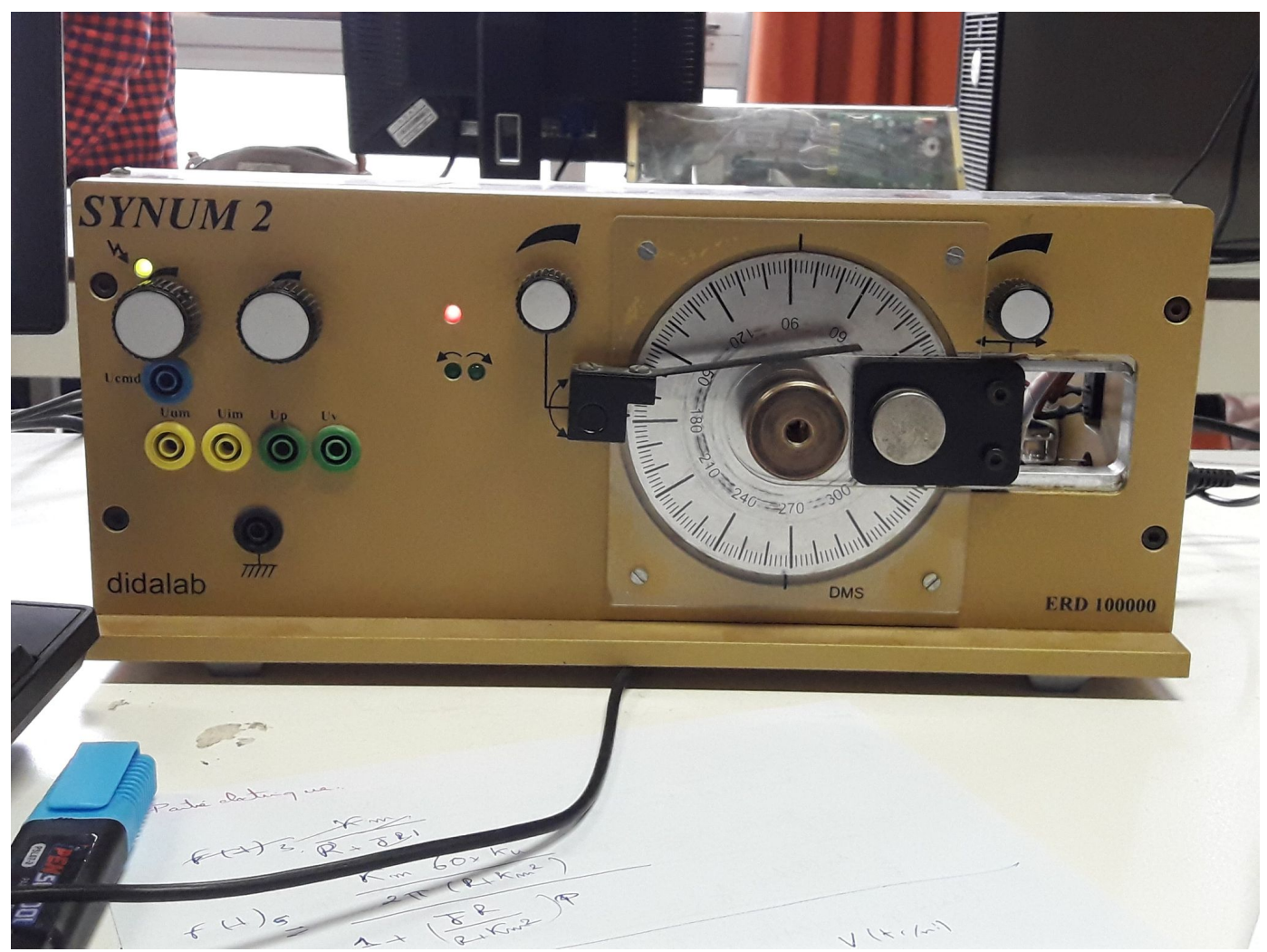

Figure 1. Le système SYNUM2 
Introduction des différentes parties du système SYNUM:

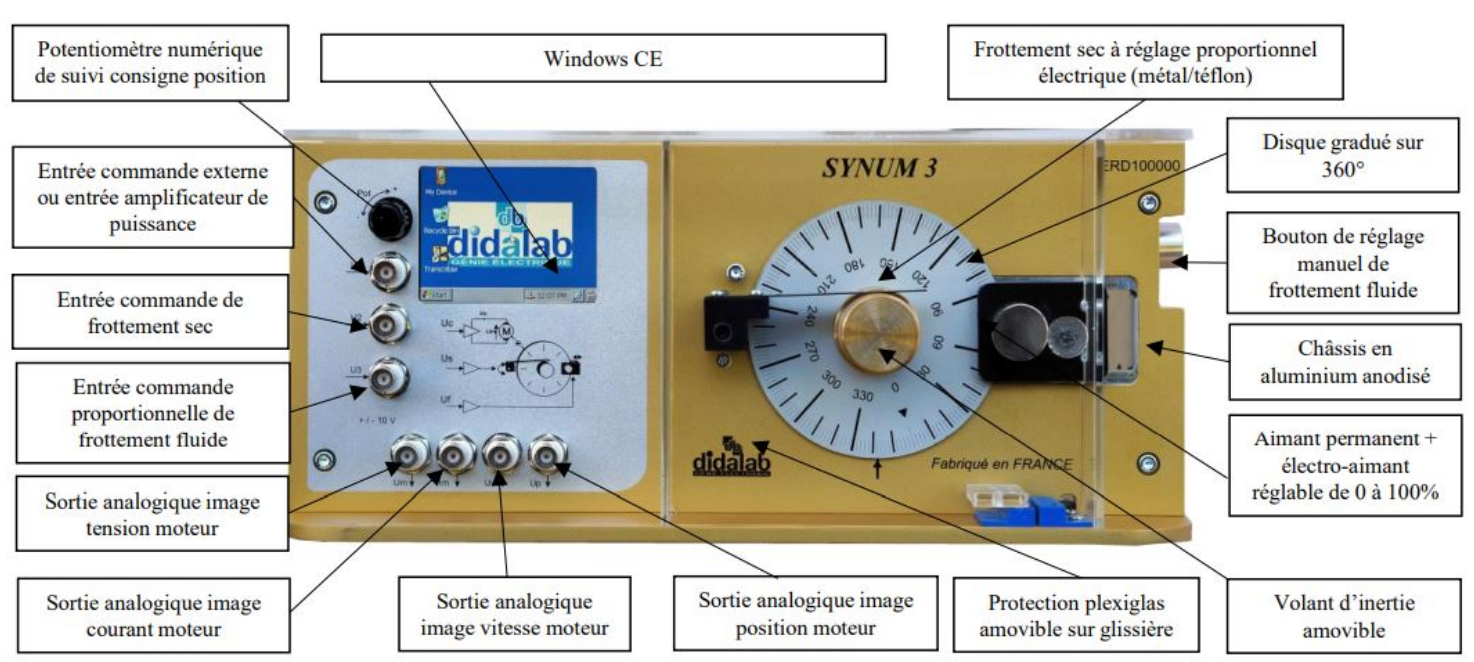

Figure 2. vue de face et les constituants du système SYNUM2

\section{Etude Théorique}

\section{Partie électrique:}

En appliquant la loi des mailles, on écrit:

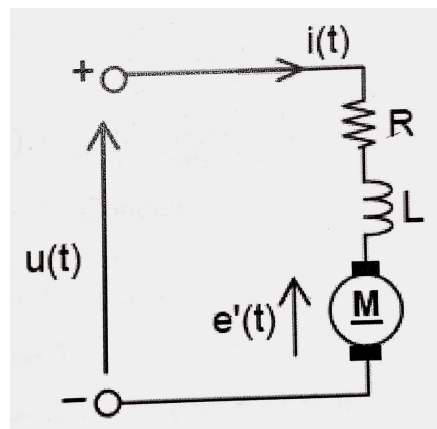

Figure 3. Circuit électrique et application de la loi des mailles

$$
u(t)=R i(t)+L \frac{d i(t)}{d t}+e_{m}(t)
$$

ainsi on lui appliquant la tarnsformée de Laplace

$$
I(p)=\frac{U(p)-E_{m}(p)}{L P+R}
$$

La Fonction de transfert de la partie électrique est:

$$
G_{e}(p)=\frac{I_{m}(p)}{U_{m}(p)-E_{m}(p)}
$$

on aura alors,

$$
G_{e}(p)=\frac{1}{R+L P}
$$


Pour identifier la constante de temps électrique $\tau_{e}$, on divise par $\mathrm{R}$ (avec $\mathrm{R}$ différente de 0 bien sûr), on aboutit ainsi à

$$
G_{e}(p)=\frac{K_{e}}{1+\tau P}
$$

avec: $K_{e}=\frac{1}{R}$ et $\tau=\frac{L}{R}$

\section{Partie mécanique:}

De manière identique au circuit électrique, on procède ici à l'application de la Relation Fondamentale de la Dynamique RFD:

$$
\sum F_{E X T}=j \frac{d \omega(t)}{d t}
$$

les deux forces à considérer sont: la force du couple moteur appliquée par la machine et notée $C_{m}(t)$ et la force de couple résistant appliquée par la charge notée $C_{r}(t)$. on aura par ailleurs,

$$
C_{m}(t)-C_{r}(t)=j \frac{d \omega(t)}{d t}
$$

avec j: le moment d'inertie des parties tournantes

en remplaçcant la force du couple résistant appliquée par la charge $C_{r}(t)$ par son expression, on aboutit à

$$
C_{m}(t)-f \omega(t)=j \frac{d \omega(t)}{d t}
$$

avec $\mathrm{f}$ le coefficient du frottement visquex, on appliquant la transformée de Laplace, on obtient finalement la Fonction de transfert de la partie mécanique

$$
G_{m}(p)=\frac{\omega(p)}{C_{m}(p)}=\frac{\omega(p)}{j P \omega(p)+f \omega(p)}=\frac{1}{f+j P}
$$

Afin de déterminer la constante de temps mécanique $\tau_{m}$, on divise par $f$, on obtient finalement une expression de la fonction de transfert mécanique contenant cette constante de temps

$$
G_{m}(p)=\frac{\left(\frac{1}{f}\right) K_{m}}{1+\left(\frac{j}{f}\right) P}=\frac{K_{m}}{1+\tau_{m} p}
$$

avec $\tau_{m}=\frac{j}{f}$ et $K_{m}=\frac{1}{f}$

Maintenant on peut facilement modéliser notre système sous la forme du schéma bloc fonctionnel ci-dessous:

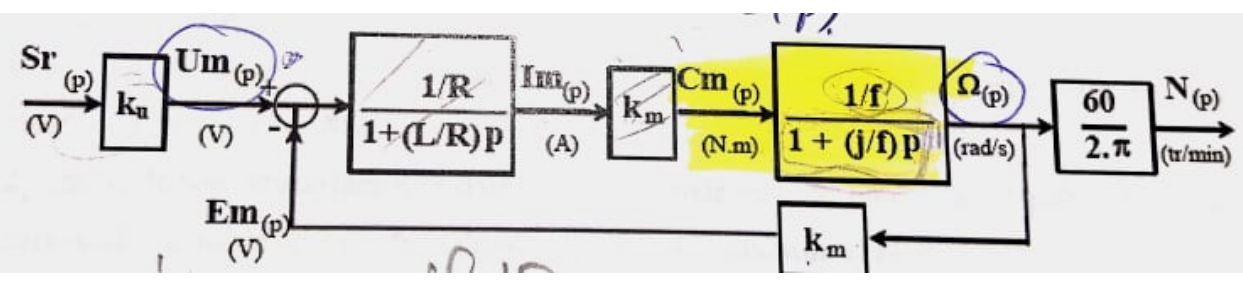

Figure 4. Le schéma bloc fonctionnel de notre système en boucle ouverte 
Pour vous faciliter la lecture de ce schéma bloc, comment procèder?

Il est plus commode de commencer la lecture du schéma bloc à partir de la sortie, c'est à dire, on commence la lecture à partir de $\omega(p)$ jusqu'à $U_{m}(p)-E_{m}(p)$.

Comme vous pouvez le constater, $\omega(p)$ est le produit de ${ }_{m}(p)$ par $C_{m}(p)$, c'est à dire le produit de $\frac{\frac{1}{f}}{1+\frac{j}{f} p} \operatorname{par} C_{m}(p)$ ce qui est conforme au résultat trouvé analytiquement.

De même, le courant $I_{m}(p)$ n'est que le produit de $\frac{\frac{1}{R}}{1+\frac{L}{R} p}$ par $\left(U_{m}(p)-E_{m}(p)\right)$, de cette façon on comprend la conception de schèma bloc fonctionnel.

Maintenant, on admet cette Hypthèse simplificatrice qui consiste à negliger la constante de temps électrique $\frac{L}{R}$, ainsi, on aura comme expression pour la fonction de transfert:

$$
F T=\frac{\frac{\frac{1}{f}}{1+\frac{j}{f} P} K_{m} \frac{1}{R}}{1+\frac{\frac{1}{f}}{1+\frac{j}{f} p} K_{m} \frac{1}{R} K_{m}}=\frac{\frac{K_{m}}{R f+K_{m}^{2}}}{1+\frac{j K}{R f+K_{m}^{2}} P}
$$

En multipliant fois $\frac{60}{2 \Pi}$, on aura comme expression finale:

$$
F T=\frac{\frac{K_{m} 60 K_{u}}{2 \Pi\left(R f+K_{m}^{2}\right.}}{1+\frac{j R}{R f+K_{m}^{2}} P}
$$

Ainsi, on obtient l'expression desirée:

$$
F T=\frac{\alpha_{2}}{1+\tau_{2} P}
$$

On désire appliqer à l'entrée une réponse à un échelon constant d'amplitude A. Alors on doit d'abord chercher la transformée de Laplace de l'amplitude A, on trouve donc:

$$
\mathcal{L}(A)=\frac{A}{p}
$$

ainsi, le comportement statique du système sera:

$$
S(t)=A \alpha_{2}\left(1-e^{\frac{-t}{\tau}}\right) U(t)
$$

lorsque

$$
t \longmapsto 0, S(t) \longmapsto 0
$$

et lorsque

$$
t \longmapsto \infty, S(t) \longmapsto A \alpha_{2}
$$


On trace ainsi la réponse indicielle suivante:

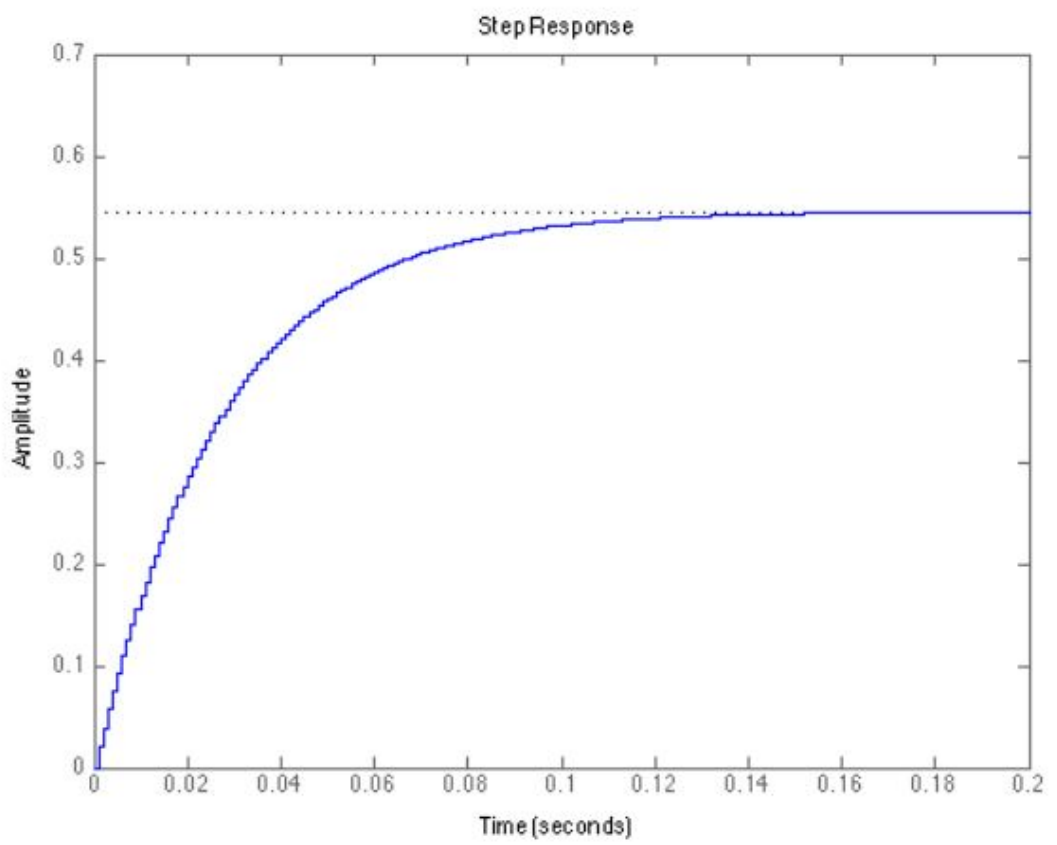

Figure 5. Réponse indicielle en boucle ouverte avec l'asymptote horizentale représente la droite d'équation $A \alpha_{2}$

On considère maintenant que le coefficient de frottement fluide est nul $(f=0)$, la nouvelle fonction de tansfert sera égale à

$$
F(t)=\frac{\frac{30 K_{u}}{\pi K_{m}}}{1+\frac{j R}{K_{m}^{2}} P}
$$

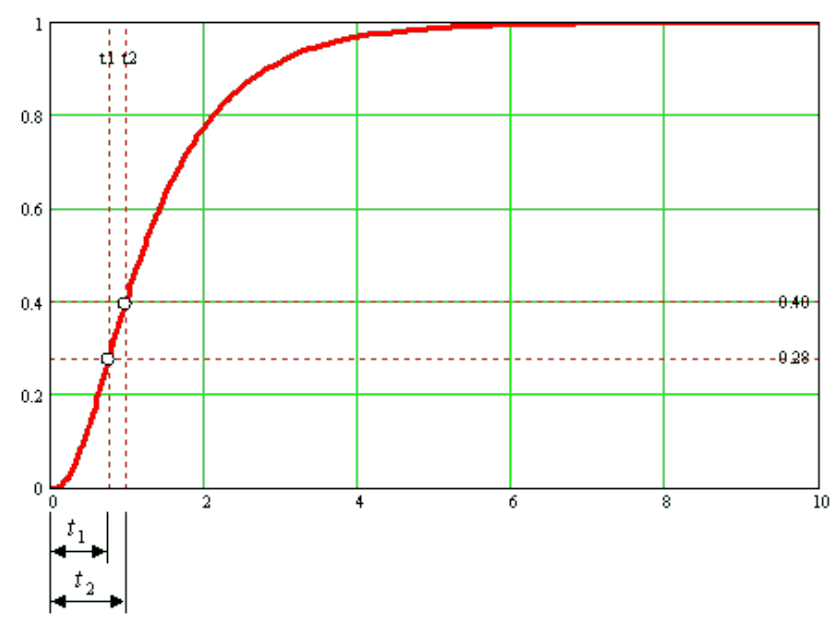

Figure 6. Réponse indicielle en boucle ouverte

Remarque: Il est à noter que l'écart entre la droite horizentale et la courbe du régime permanent ne réprésente pas l'erreur vu qu'on opére en boucle ouverte et qu'il n'ya pas une consigne d'entrée 


\section{Etude expérimentale}

\section{Etude en régime dynamique}

- On configure notre système

- $\quad$ on choisit comme tension de repos égale à $0 \mathrm{~V}$

1. On choisit la commande "Echelon constant" avec une valeur égale à $10 \mathrm{~V}$ et un retard de $0,2 \mathrm{~s}$

2. on sélectionne les points d'enregistrement $S_{r}$ et $N_{m}$ en cliquant dessus

3. on applique la commande en cliquant sur le commutateur

4. on visualise les courbes de réponses obtenues.

- On ajuste les échelles afin d'obtenir des allures satisfaisantes.

- $\quad$ on relève le temps de réponse à $5 \%$

- $\quad$ On refait la même procedure avec un frottement fluide à 100 ci-dessous les valeurs tabulées:

\section{En absence de frottement:}

\begin{tabular}{ccccccccc}
\hline \hline$S_{r}$ & -20 & -15 & -10 & -5 & 5 & 10 & 15 & 20 \\
\hline$N_{m}(t r / \min )$ & -4372 & -3230 & -2106 & -1006 & 1014 & 2110 & 3236 & 4335 \\
$U_{m}(V)$ & $-21,9$ & $-16,4$ & $-10,6$ & -5 & 5 & 10,4 & 16,4 & 21,6 \\
\hline
\end{tabular}

Table 1: Valeurs tabulées des vitesses et Tensions en absence de frottement fluide

En présence de frottement à $100 \%$ :

\begin{tabular}{ccccccccc}
\hline \hline$S_{r}$ & -20 & -15 & -10 & -5 & 5 & 10 & 15 & 20 \\
\hline$N_{m}(t r / \min )$ & -1997 & -1497 & -977 & -462 & 472 & 948 & 1505 & 2010 \\
$U_{m}(V)$ & -10 & $-11,9$ & $-7,9$ & $-3,9$ & 3,8 & 7,9 & 12,1 & 16 \\
\hline
\end{tabular}

Table 2: Valeurs tabulées des vitesses et Tensions en presénce de frottement fluide

On procède maintenant aux calculs de la constante de temps $\tau$ et du temps de réponse à $5 \%$ dans le cas de l'absence de frottement

On trouve alors: $\tau=0,3585 \mathrm{~s}$ et $t_{r 5 \%}=2,890 \mathrm{~s}$

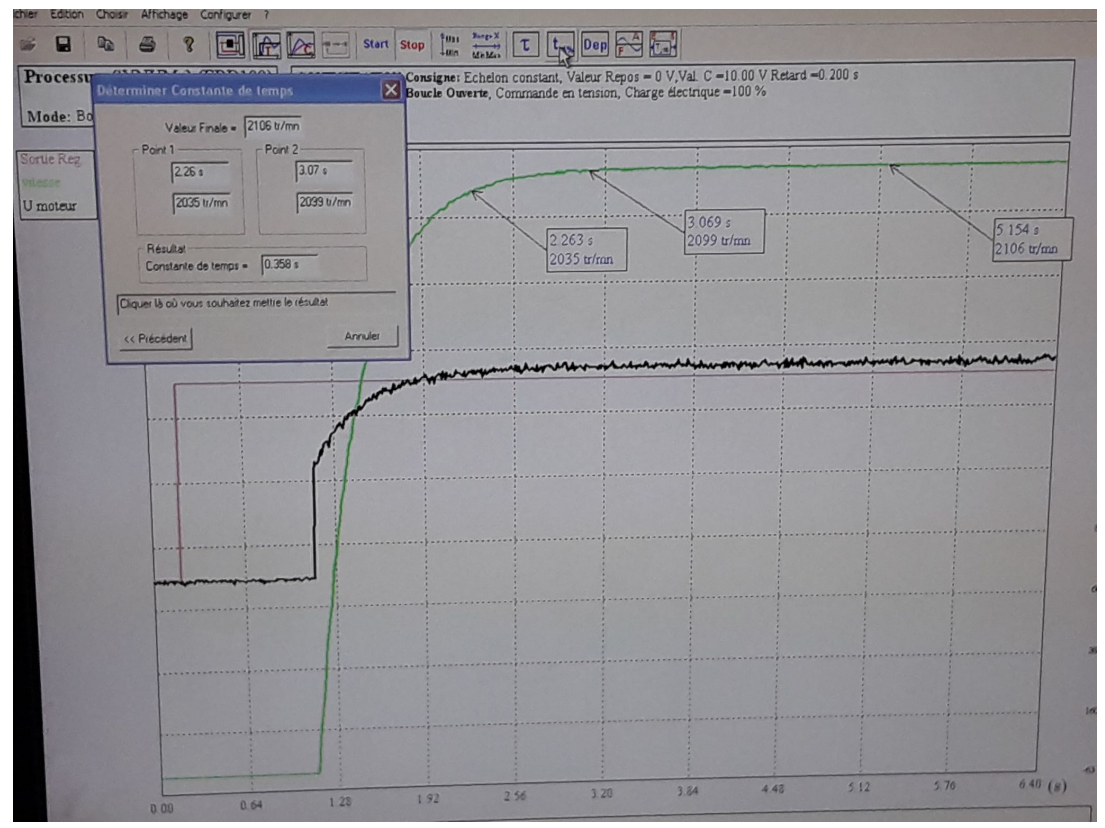

Figure 7. Calcul des temps de réponse et constante de temps 
Dans le cas où il n'ya pas de frottement, on trace les courbes $U_{m}=f\left(S_{r}\right)$ et $N_{m}=f\left(S_{r}\right)$ en utilisant MATLAB

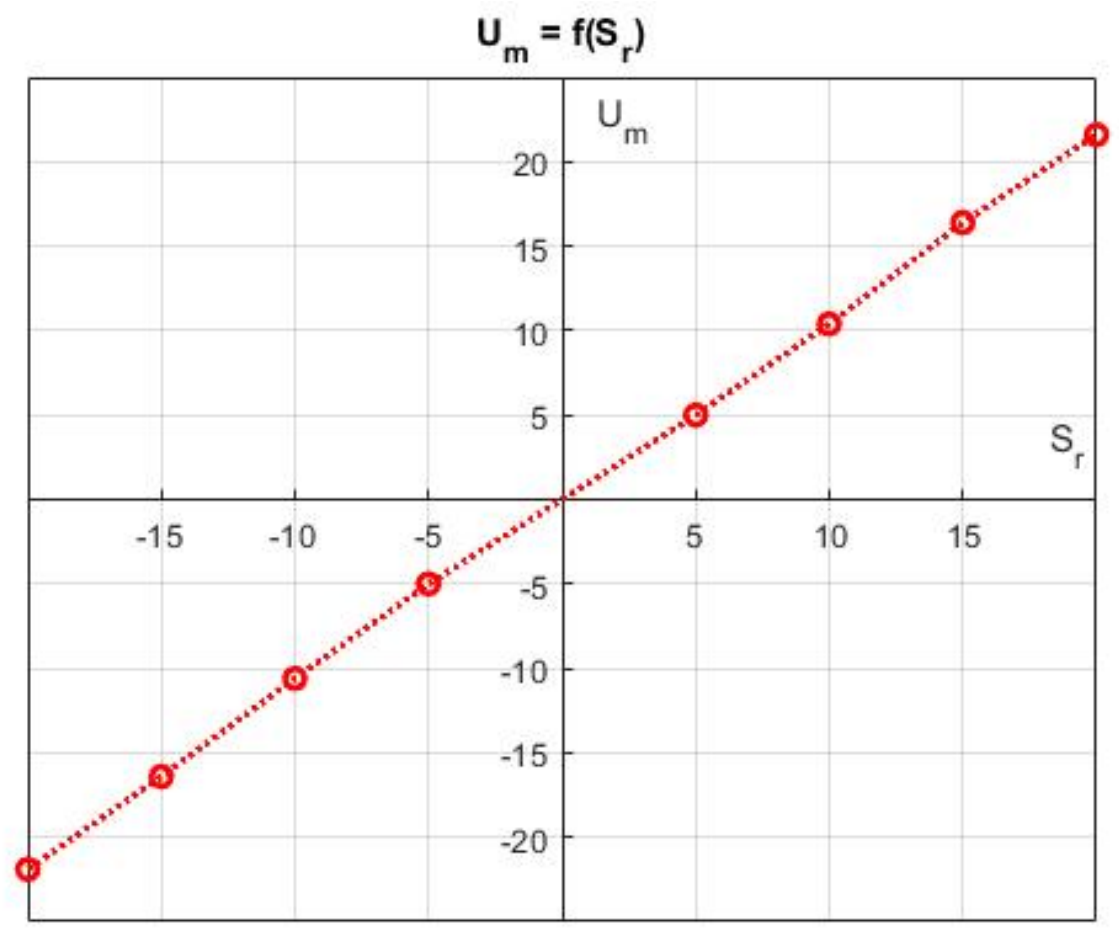

Figure 8. la courbe $U_{m}=f\left(S_{r}\right)$

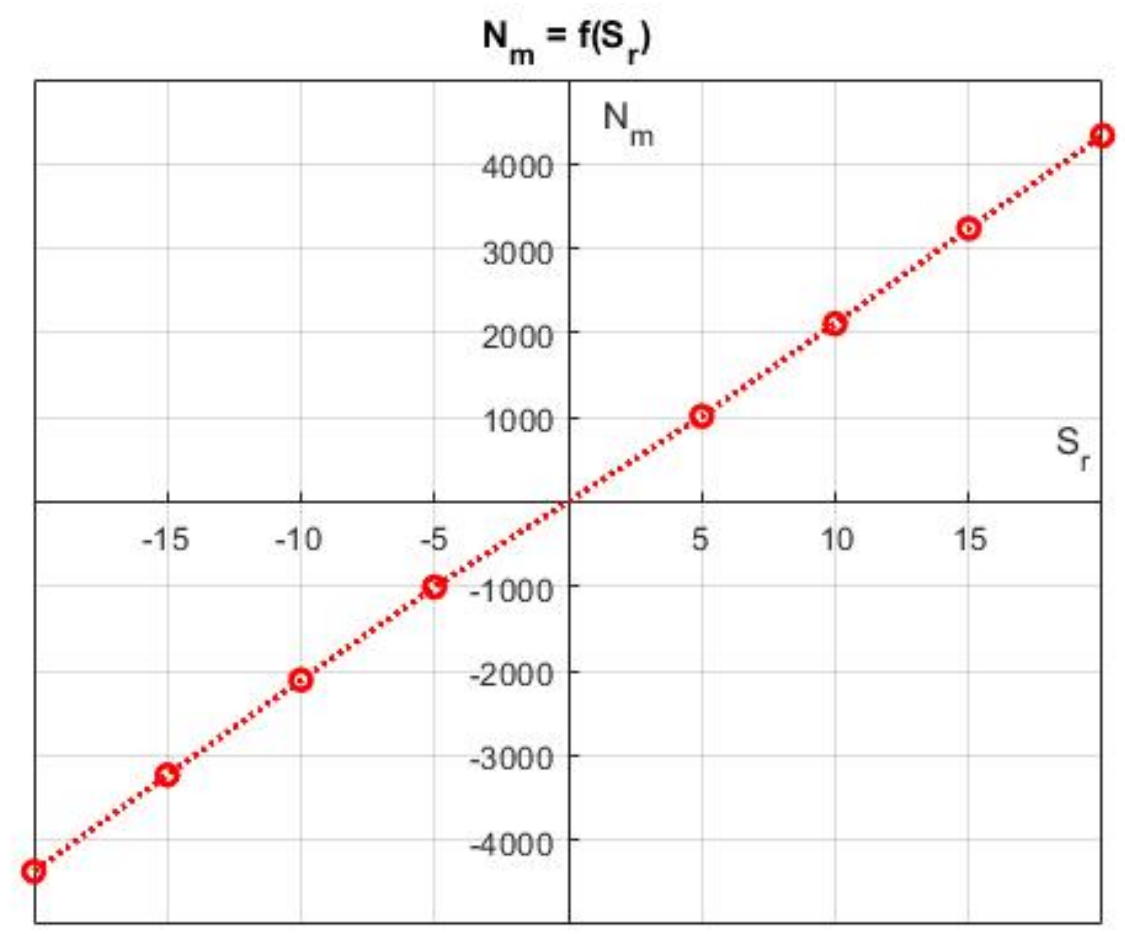

Figure 9. la courbe $N_{m}=f\left(S_{r}\right)$ 
On procède maintenant aux caluls des pentes des deux courbes, on trouve:

Pente $_{1}=1.1$

Pente $_{2}=228,4$

Voici les codes MATLAB utilisés pour tracer les deux courbes ci-dessus:

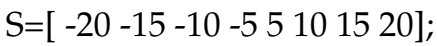

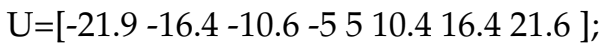

$\mathrm{N}=[-4372$-3230 -2106 -1006 101421103236 4335];

$\mathrm{h}=$ figure ();

plot(S,U,':or','LineWidth',2);

set(gca, 'XAxisLocation', 'origin', 'YAxisLocation', 'origin')

xlabel (' $S_{r}$ ')

ylabel (' $\left.U_{m}{ }^{\prime}\right)$

title ('U $\left.U_{m}=f\left(S_{r}\right)^{\prime}\right)$

grid on

hold on

plot(S,N,':or','LineWidth', 2);

set(gca, 'XAxisLocation', 'origin', 'YAxisLocation', 'origin')

xlabel ( ' $S_{r}$ ')

ylabel (' $\left.N_{m}{ }^{\prime}\right)$

title ('N $\left.N_{m}=f\left(S_{r}\right)^{\prime}\right)$

grid on

hold on

\section{Conclusion}

Elargissions les horizons,

Quelles sont les performances d'un système d'asservissement ?

Plusieurs paramètres caractérisent les performances d'un asservissement :

- la rapidité se définit par la vitesse à laquelle une valeur stable en sortie est atteinte. C'est le temps de réponse ;

- la stabilité. Pour une entrée constante, un système est stable si la sortie tend vers une valeur finie. Si elle oscille ou diverge, l'asservissement est instable ;

- le dépassement de la consigne ; souvent exprimé en \% d'erreur. Même lorsqu'un système est stable, il arrive que la sortie dépasse la consigne avant de se stabiliser, ce qui n'est pas nécessairement une anomalie ;

- la précision, c'est-à-dire la capacité de l'asservissement à atteindre la consigne avec précision le plus rapidement possible.

Un asservissement agit sur une ou plusieurs caractéristiques de la grandeur asservie telles que :

- la position : asservissement de position ;

- la vitesse : asservissement de vitesse ;

- l'accélération : asservissement d'accélération.

La stabilité et la précision étant souvent antagonistes de la rapidité, pour améliorer ces caractéristiques, il faut modifier les caractéristiques internes du système ou ajouter un correcteur dans la boucle d'asservissement. Il existe différents types de correcteurs, leur action peut être intégrale, dérivée et/ou proportionnelle. 
Author Contributions: LABIDI Aymen, please turn to my Research gate and Linkedin for the term explanation and more scientific papers.

Institutional Review Board Statement: National Institute of Applied Sciences and Technology, Tunis, Tunisia, Chemical Engineering Student, Chemical Engineering Department - Organic ChemistryOrgano-metallic Chemistry - Analytical Chemistry

Sample Availability: Samples and more researches and papers are available from author'sResearch gate profile
Abbreviations
The following abbreviations are used in this article:
$C_{m} \quad$ Couple moteur appliqué par la machine
$C_{r} \quad$ Couple moteur appliqué par la charge
j Moment d'inertie
$\omega(t) \quad$ Vitesse angulaire de rotation
$\frac{d \omega(t)}{d t} \quad$ Accéleration angulaire
$f \quad$ Coefficient de frottement

\section{Appendix G Other sources}

to learn more about Mechanical, Electrical and Electrotechnic engineering, you can search for articles in Techniques de l'ingénieurs

\section{References}

1. J.-M. Pierrel, J. Dendien and P. Bernard, Actes de EURALEX, 2004, 4, year. 
